\title{
Caracterización por XANES, análisis mineralógico y aplicación industrial de un depósito de caolín de México
}

\section{A Mexican kaolin deposit: XANES characterization, mineralogical phase analysis and applications}

\author{
F. Vázquez ${ }^{(*)}$, L. M. Torres ${ }^{(* *)}$, L. L. Garza(**) A. Martínez ${ }^{(*)}$ y W. López ${ }^{(* *)}$
}

Recepción/Received: 12-IX-07

Aceptación/Accepted: 12-IV-08

Publicado online/Online publishing: 22-V-09

\section{RESUMEN}

El caolín obtenido de Villa de Reyes, una región cercana a San Luis Potosí, México, fue caracterizado por las siguientes técnicas: difracción de rayos- $X$ en polvos (DRX), microscopía óptica (MO), microscopía electrónica de barrido (MEB), fluorescencia de rayos-X (FRX), espectroscopía de absorción de rayos-X (XANES), análisis térmico (DTA/TGA), dilatometría (DIL) y análisis químico. Los resultados del análisis mineralógico mediante DRX mostraron un mineral constituido principalmente de caolinita, con una contribución minoritaria de cuarzo, cristobalita, tridimita y dolomita. El análisis por microscopía óptica reveló un alto contenido de material amorfo volcánico, indicando una caolinización incompleta del material vítreo. El color rojo observado en el caolín fue asociado con la presencia de hierro en la forma de limonita $[\mathrm{FeO}(\mathrm{OH})]$, la cual fue determinada mediante XANES. Se analizó la influencia del tamaño de partícula en el color del caolín. El análisis dilatométrico reveló una fuerte expansión térmica entre 110 y 240 ${ }^{\circ} \mathrm{C}$, lo cual dificultaría el empleo del material estudiado en la industria cerámica tradicional, en cambio la presencia de vidrio y las fases de alta temperatura del $\mathrm{SiO}_{2}$, cristobalita y tridimita, favorecen su empleo en la industria cementera.

Palabras clave: caolín, caracterización, minerales, cerámica, SEM.

\section{SUMMARY}

A kaolin obtained from Villa de Reyes, a region near to San Luis Potosí (México) was characterized by means of $X$-ray powder diffraction (XRD, optical microscopy (OM), scanning electron microscopy (SEM), X-ray fluorescence (XRF), $X$-Ray Absorption Near Edge Spectroscopy (XANES), thermal analysis (DTA/TGA), dilatometry (DIL), and chemical analysis. Mineralogical and morphological characteristics of the mineral are presented. The kaolin sample was formed mainly by kaolinite, but other minor phases were also detected such as quartz, cristobalite, tridymite, and dolomite. The high content of volcanic glass detected, by optical microscopy, revealed an incomplete kaolinization process of the raw material. The reddish color of the kaolin was associated with the free iron content in the form of limonite $[\mathrm{FeO}(\mathrm{OH})]$, which was determined by XANES. The influence of the particle size on the whiteness of kaolin was evaluated. Dilatometric analysis revealed a strong thermal expansion between 110 y $240^{\circ} \mathrm{C}$, which would difficult the use of this material in traditional ceramic applications. On the other hand the presence of glass and high temperature phases of $\mathrm{SiO}_{2}$, such as cristobalite and tridymite will favor its use in the cement industry.

Keywords: kaolin, characterization, minerals, ceramic, SEM.

(*) Universidad Autónoma del Estado de Morelos, Cuernavaca (México).

(**) Universidad Autónoma de Nuevo León, N. L. (México).

(***) CEMEX, Cemex Technical Center (USA). 


\section{INTRODUCCIÓN}

En la actualidad, el caolín es uno de los minerales más utilizados en la industria cerámica como materia prima dadas sus diversas aplicaciones en artículos de cerámica, refractarios, cemento, fibra de vidrio, etc., además es utilizado ampliamente en otras industrias como la de pintura, papel, pesticidas, farmacéuticos y cosméticos (1). En términos generales, se denomina caolín al mineral con alto contenido de caolinita. Los principales yacimientos de este mineral alrededor del mundo se encuentran localizados en el sureste de Estados Unidos, Brasil, Francia, Reino Unido, Alemania y China. En este sentido, dado el potencial de su aplicación industrial, diversos caolines provenientes de distintas regiones del mundo han sido caracterizados (2-4).

Frecuentemente los minerales extraídos de la superficie terrestre presentan grados altos de heterogeneidad debido a la presencia de las impurezas que los acompañan. Dependiendo de las condiciones atmosféricas y geológicas de la región, así como del grado de alteración del entorno de donde se extrae la arcilla, impurezas tales como hematita, limonita, ilmenita, pirita, magnetita, cromita, etc., suelen estar presentes. Estos minerales aportan un número importante de elementos cromóforos, además del $\mathrm{Fe}$ y $\mathrm{Ti}$, tales como $\mathrm{Cr}$, Mn y Ni los cuales presentan la propiedad de pigmentar la materia prima $y$, por ende, el producto final procesado $(5,6)$.

El contenido de estos elementos cromóforos en las materias primas juega un papel muy importante en los procesos industriales, dado que pequeñas variaciones por encima de la concentración permisible de éstos conduce a alteraciones significativas en la pigmentación del producto final. En México, éste es uno de los principales problemas a resolver en la industria cerámica, especialmente en la producción de cemento blanco. Para la solución de este problema, diferentes técnicas físicas, químicas y microbiológicas han sido propuestas para la eliminación de hierro presente en arcillas, y particularmente en caolines. Así, algunos trabajos previos han abordado la problemática mediante el uso de separaciones físicas (magnética o por tamices) $(7,8)$, por adsorción física mediante ceniza de cáscara de arroz $(9)$, métodos de floculación $(10,11)$ y tratamiento químico con ácido clorhídrico, hidrosulfito de sodio, ditionato de sodio, etc. (9-12). No obstante, es importante hacer mención que el empleo de estas técnicas no ha conducido a la obtención de resultados del todo satisfactorios. Es por lo anterior que en los últimos años se ha manifestado una importante tendencia hacia el estudio del caolín y su proceso de blanqueo. Algunas de estas investigaciones apuestan en la dirección del uso de agentes quelantes orgánicos como los ácidos: oxálico, malónico y cítrico, el EDTA y la tiourea (13-15) que actúan como secuestrantes de hierro para eliminar su coloración.

\section{OBJETIVO}

El objetivo del presente trabajo es la caracterización de un caolín proveniente de la región de Villa de Reyes, en San Luis Potosí, México. Evaluar el contenido en hierro (Fe) y su naturaleza dentro del caolín con el fin de determinar su posible aplicación industrial.

\section{DESARROLLO EXPERIMENTAL}

El mineral analizado en el presente trabajo es un caolín rojizo originario del graben de Villa de Reyes, que se encuentra localizada en San Luis Potosí, México. Este graben fue creado en el Oligoceno hace 28 millones de años, es un

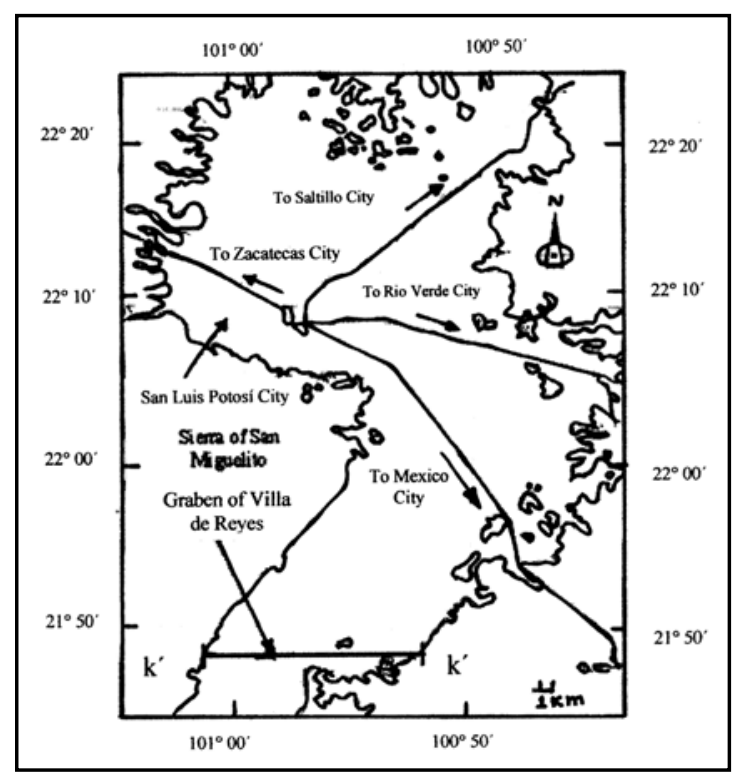

Figura 1. Localización del graben de Villa de Reyes, San Luis Potosí, México. 
valle tectónico hundido, limitado por fallas normales y relleno por el miembro inferior de la riolita Panalillo y por materiales de acarreo. Geográficamente, la región se encuentra situada en $15 \mathrm{~N} 40^{\circ}$ inclinada hacia $\mathrm{N} 10^{\circ} \mathrm{E}$ como se observa en la Figura 1. El graben tiene una extensión de 260 km de largo por $14 \mathrm{~km}$ de ancho.

Como tratamiento inicial, el caolín obtenido en greña de la mina fue secado, triturado y molido por espacio de 90 minutos en un molino de porcelana con bolas de alúmina de alta densidad, con el fin de reducir su tamaño de partícula y de homogeneizar el material. La molienda del caolín fue efectuada hasta obtener un residuo de $10 \%$ en malla 200 , esto es, el $90 \%$ del material tamizado tuvo un tamaño de partícula menor a $75 \mu \mathrm{m}$. El efecto del tamaño de partícula en el color del caolín fue evaluado mediante el estudio de la propiedad óptica en función del tiempo de molienda, proceso que se llevó acabo en un molino de zirconio. Los parámetros de color $L^{*}, a^{*}, b^{*}$ y el grado de blancura CIE Ganz 82 fueron medidos a diferentes tiempos de molienda.

El hierro libre contenido en el caolín rojizo fue removido mediante un proceso de lixiviación de $3 \mathrm{~g}$ de mineral con 20 $\mathrm{ml}$ de $\mathrm{HCl}$ concentrado a $50{ }^{\circ} \mathrm{C}$ por 30 minutos. El material resultante fue sucesivamente lavado con agua destilada, secado y almacenado para su posterior análisis. El análisis mineralógico de la muestra inicial fue realizado a través de diversas técnicas de caracterización como difracción de rayos-X en polvo (DRX), microscopía óptica (MO), microscopía electrónica de barrido (SEM), fluorescencia de rayos-X (FRX), espectroscopía de absorción de rayos-X (XANES), análisis térmico (DTA/TGA) y análisis químico. El análisis dilatométrico (DIL) se realizó sobre una pieza quemada de $5 \mathrm{~cm}$ de longitud y $7 \mathrm{~mm}$ de diámetro, la cual fue preparada previamente mediante prensado en seco y cocción en un horno industrial de rodillos a $1.150^{\circ} \mathrm{C}$ con un ciclo de 41 minutos.

\section{RESULTADOS Y DISCUSIÓN}

Como producto de la molienda del caolín, se obtuvo un polvo fino de coloración rojiza. El color del material pulverizado fue medido en el sistema internacional CIE L*a*b*. En primera instancia, el color observado en la muestra está asociado con el contenido en hierro $\left(1,49 \% \mathrm{Fe}_{2} \mathrm{O}_{3}\right)$ de acuerdo al análisis químico del mineral realizado por fluorescencia de rayos-X (Tabla 1 ). En la tabla se presentan los resultados del análisis de la composición química del caolín en forma de óxidos.

El análisis por difracción de rayos- $X$ reveló la naturaleza de las fases cristalinas presentes en el mineral. La muestra estudiada está constituida en mayor proporción por el mineral caolinita (JCPDS 01-078-1996), de acuerdo

Tabla 1

Composición química del mineral determinado por FRX.

\begin{tabular}{|c|c|}
\hline Óxido & Cantidad total (\%) \\
\hline $\mathrm{SiO}_{2}$ & 72.63 \\
\hline $\mathrm{Al}_{2} \mathrm{O}_{\mathbf{3}}$ & 15.04 \\
\hline $\mathrm{Fe}_{\mathbf{2}} \mathrm{O}_{3}$ & 1.49 \\
\hline $\mathrm{CaO}$ & 0.70 \\
\hline $\mathrm{MgO}$ & 0.55 \\
\hline $\mathrm{K}_{\mathbf{2}} \mathrm{O}$ & 0.65 \\
\hline $\mathrm{TiO}_{\mathbf{2}}$ & 0.34 \\
\hline $\mathrm{PPI} 950^{\circ} \mathrm{C}$ & 8.25 \\
\hline $\mathrm{TOTAL}^{\circ}$ & 99.96 \\
\hline
\end{tabular}

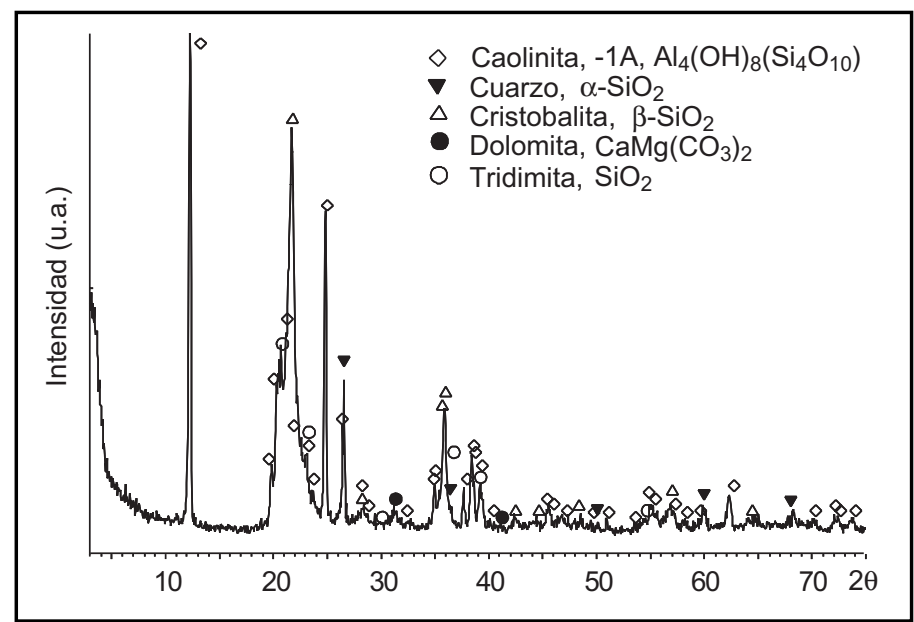

Figura 2. Patrón de difracción de rayos-X del caolín proveniente del graben de Villa de Reyes. 
con la presencia de sus principales líneas de difracción a 7,15 $\AA$ (001) y $3,58 \AA$ (002). Asimismo, fueron detectadas contribuciones menores de fases cristalinas, como cuarzo (JCPDS 03-065-0466), cristobalita (JCPDS 01082-0512), tridimita (JCPDS 01-071-0197) y dolomita (JCPDS 01-073-2324) (Figura 2). De las fases cristalinas detectadas, dado el bajo contenido en hierro que arrojó el análisis químico, ninguna incluyó a este elemento en su composición. Por esta razón, se emplearon técnicas adicionales de caracterización con el fin de determinar la presencia de compuestos de hierro y el entorno químico del elemento dentro del mineral.
Mg y $\mathrm{K}$ en niveles de trazas. El análisis químico mostró un alto contenido de $\mathrm{SiO}_{2}$, respecto al contenido de $\mathrm{Al}_{2} \mathrm{O}_{3}$, lo cual es típico de un material pobremente caolinizado.

Para determinar la presencia minoritaria de fases cristalinas en el mineral, principalmente aquéllas de hierro, se recurrió al análisis por microscopía óptica de sección delgada. Mediante esta técnica fue posible determinar que el mineral analizado corresponde a una toba vítrea caolinizada, la cual presenta un alto contenido vítreo y la presencia minoritaria de hematita, feldespato y fenocristales de sílice. De acuerdo a lo observado, los pequeños filamentos

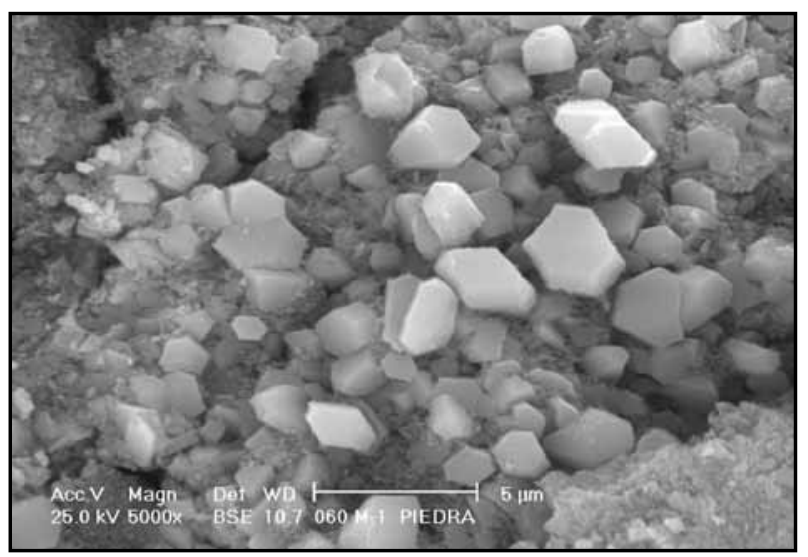

Figura 3. Micrografía tomada por microscopía electrónica de barrido del caolín estudiado.

La morfología del mineral fue analizada mediante la técnica de SEM. La principal característica observada fue la presencia de cristales de geometría hexagonal con dimensiones de 1 a $4 \mu \mathrm{m}$, como se puede observar en la Figura 3. De acuerdo a lo encontrado en la literatura (16), esta geometría corresponde con la presencia de cristales de caolinita. El análisis por EDS determinó que los principales constituyentes del mineral son $\mathrm{O}, \mathrm{Si}$ y $\mathrm{Al}$; con la presencia de rojos absorbidos en la superficie del caolín pueden ser de compuestos de hierro, como el óxido e hidróxido. La micrografía de la Figura 4a muestra una zona clara debido a la presencia de material vítreo, asociado con el proceso de formación del mineral. El proceso de transformación de la toba volcánica, de fase vítrea a la cristalización de caolinita, puede considerarse incompleto debido probablemente a la escasa presencia de fluidos o a la baja temperatura

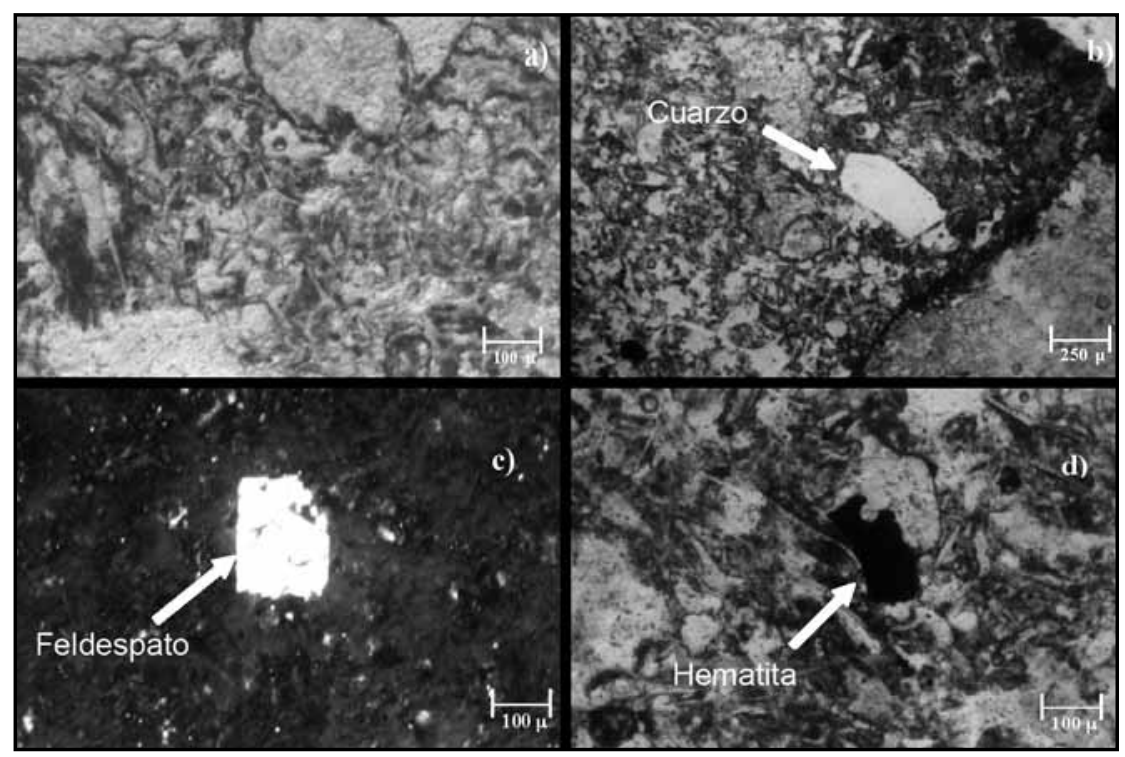

Figura 4. Diferentes regiones de una muestra de caolín analizada por microscopía óptica. 
presente durante el proceso hidrotérmico que dio origen a la caolinización del mineral durante la actividad volcánica. La micrografía de la Figura $4 b$ muestra un fenocristal de cuarzo de $250 \mu \mathrm{m}$. Al igual que en la micrografía anterior, nuevamente se observa un alto contenido vítreo en el mineral analizado. En la micrografía de la Figura 4c, tomada a 100X con luz polarizada, se observa en el centro de la misma un área brillante correspondiente a un cristal de feldespato de aproximadamente $100 \mu \mathrm{m}$. Asimismo, en el centro de la Figura 4d se puede apreciar uno de los escasos fenocristales de hematita presentes en la muestra.

Tomando en cuenta los resultados anteriores, es posible concluir que el mineral analizado posee un alto contenido vítreo. La cantidad de $\mathrm{SiO}_{2}$ presente en el mineral (72,63\%) excede en gran proporción la cantidad estequiométrica requerida (18\%) para reaccionar completamente con el $\mathrm{Al}_{2} \mathrm{O}_{3}(15,05 \%)$ y formar caolinita pura. De igual manera, la presencia de algunos fenocristales de cuarzo no es suficiente para justificar el alto contenido de cuarzo, por lo que dicho excedente se encuentra principalmente formando parte del vidrio y no como $\mathrm{SiO}_{2}$ libre.

El color rojo observado en el caolín es debido principalmente a la presencia de filamentos rojos adsorbidos en la superficie de la caolinita. Se realizó un análisis por XANES al mineral con el fin de determinar la naturaleza de los compuestos de hierro presentes. Para este propósito, el espectro XANES de diversos compuestos estándar de hierro como $\mathrm{FeO}, \mathrm{Fe}_{3} \mathrm{O}_{4}, \mathrm{FeSO}_{4}$, $\mathrm{FeO}(\mathrm{OH}), \mathrm{FeS}, \mathrm{FeSe}$, y $\mathrm{ZnFeO}_{4}$ fueron tomados como referencia. En base a lo anterior se determinó una buena correlación entre el espectro XANES del caolín y el correspondiente a $\mathrm{FeO}(\mathrm{OH})$ (Figura 5). Teniendo en cuenta este resultado y considerando que el espectro XANES representa la huella digital de un compuesto dado, se puede concluir que el hierro presente dentro del mineral estudiado existe en la forma de limonita $[\mathrm{FeO}(\mathrm{OH})]$. Para determinar la distribución del Fe dentro de la fases vítrea y/o caolinítica, y fuera de ellas, el mineral fue sometido a un proceso de lixiviación con $\mathrm{HCl}$ con el fin de eliminar el hierro libre, esto es, aquél que se encontraba fuera de las fases caolinita y vítrea. Como producto del proceso de lixiviación fue obtenido un material de color blanco. El análisis químico por FRX reveló una reducción en el contenido en hierro de 1,49 a $0,45 \%$ expresado en forma de $\mathrm{Fe}_{2} \mathrm{O}_{3}$. La cantidad total de la misma especie química presente en el caolín, determinada por espectroscopía de absorción atómica fue de 1,46\%, valor muy similar al obtenido por fluorescencia de rayos-X (Tabla 1$)$.

Bajo la consideración de que sólo el hierro libre puede ser removido en el proceso de lixiviación, es posible concluir que el $70 \%$ del hierro presente en el mineral se encuentra libre y no formando parte de las fases cristalinas, ni vítreas del material.

La variación de los parámetros de color $L^{*}, a^{*}, b^{*}$ y el grado de blancura CIE Ganz 82 del caolín fue evaluada en función del tiempo de molienda. El proceso anterior se realizó en el caolín molido inicialmente a malla 200, así como en el sometido al proceso de lixiviación. Como se puede observar en la Figura 6, luego de 20 minutos de molienda se observa una estabilidad en los parámetros de color (Tabla 2). Durante este periodo de tiempo, el tamaño de caolín fue reducido de 21,7 a 3,6 $\mu \mathrm{m}$. De acuerdo a lo anterior, el caolín sin tratar se tornó más rojizo, lo cual indica que el $\mathrm{FeO}(\mathrm{OH})$ tiene propiedades pigmentantes. Un fenómeno totalmente opuesto ocurrió en la muestra sometida al proceso de lixiviación con $\mathrm{HCl}$ (Tabla 2). Lo anterior confirma que el hierro libre es la especie química responsable de la tonalidad rojiza del caolín.

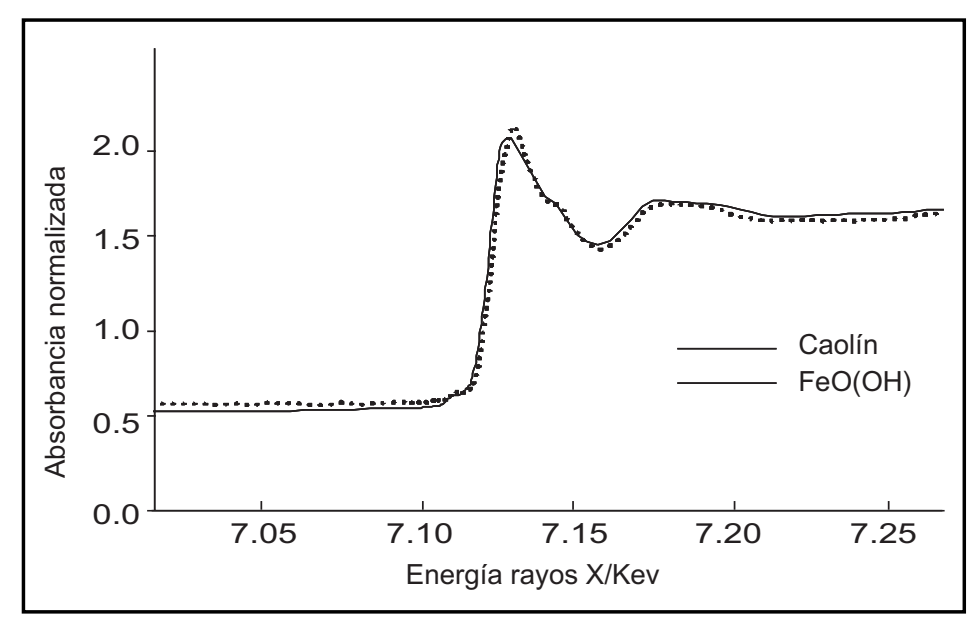

Figura 5. Espectro XANES del caolín superpuesto al espectro XANES del FeO(OH). 


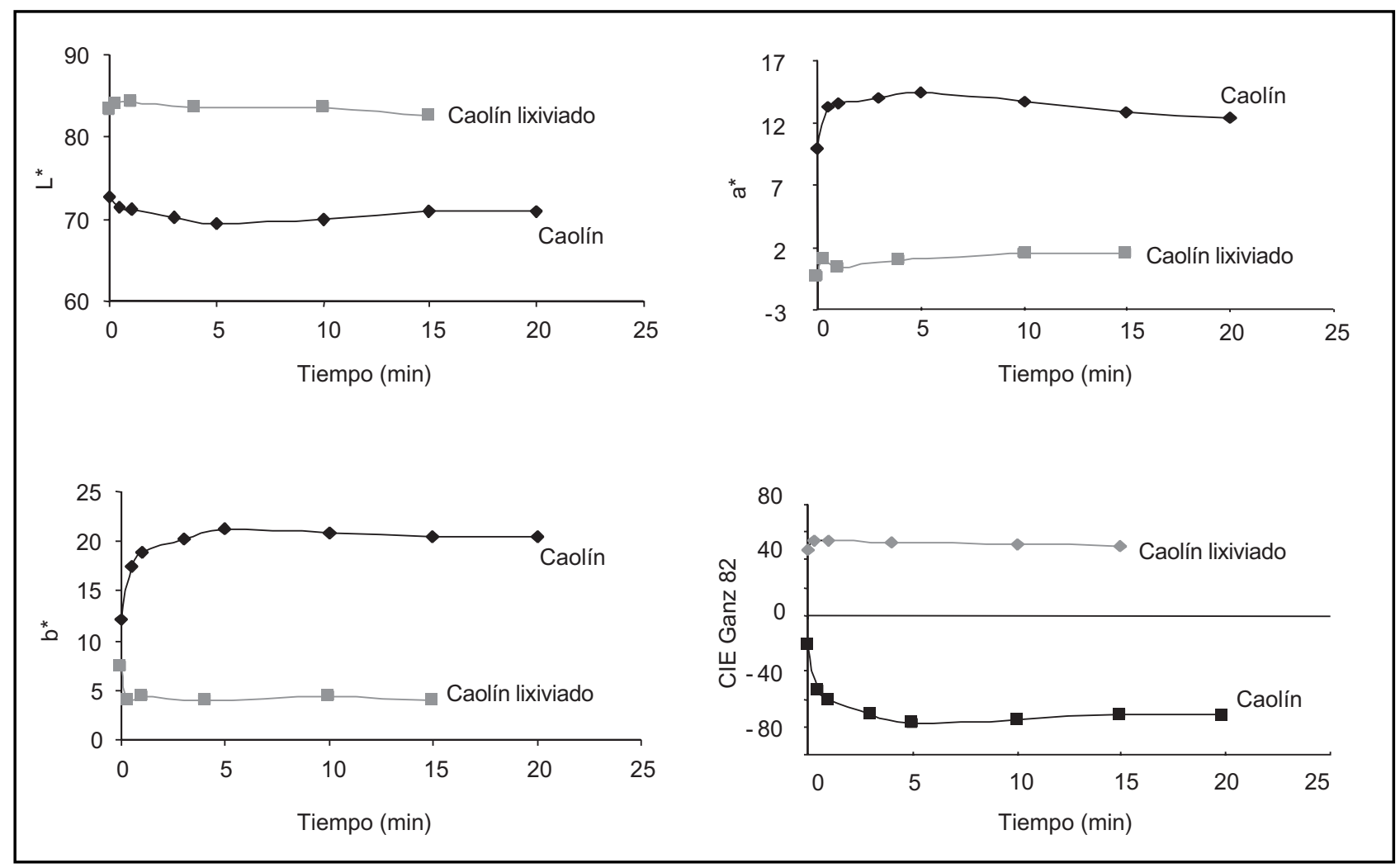

Figura 6. Evolución de los parámetros de color $L^{*}, a^{*}, b^{*}$ y blancura del caolín en función del tiempo de molienda.

Tabla 2

Parámetros de color y tamaño de partículas del material nativo, molido y lixiviado.

\begin{tabular}{|c|c|c|c|c|c|}
\hline Muestra & $\begin{array}{c}\text { Tamaño partícula } \\
(\mathbf{\mu m})\end{array}$ & $\mathbf{L}^{*}$ & a* & b* & CIE Ganz 82 \\
\hline Caolín antes de la lixiviación, sin molienda & 21.7 & 74.25 & 10.72 & 13.3 & -23.33 \\
\hline Caolín antes de la lixiviación, con molienda & 3.6 & 71.03 & 12.42 & 20.43 & -70.65 \\
\hline Caolín después de la lixiviación, sin molienda & - & 84.80 & 0.24 & 3.70 & 47.80 \\
\hline
\end{tabular}

La curva de DTA del mineral analizado mostró una serie de picos endotérmicos y exotérmicos (Figura 7). Los dos picos endotérmicos observados en el intervalo de temperatura de $25-250{ }^{\circ} \mathrm{C}$ corresponden a la pérdida de agua adsorbida y ocluida en el mineral. De la curva de TGA se estimó una pérdida en peso total del $4 \%$ debido a estos procesos. El tercer pico observado a $220{ }^{\circ} \mathrm{C}$ corresponde con un proceso exotérmico que puede estar asociado con la transición cristalina de $\alpha$ a $\beta$, cristobalita. En el intervalo de temperatura de 400$700{ }^{\circ} \mathrm{C}$ se puede apreciar la presencia de tres picos endotérmicos. El primero corresponde a la transición cristalina del cuarzo de su forma $\alpha$ a $\beta$ ( $\left.573^{\circ} \mathrm{C}\right)$; el segundo pico es acompañado de una pérdida de masa debido a la eliminación de $\mathrm{OH}$ unidos a la caolinita (deshidroxilación). El tercer pico puede ser asociado a la presencia de fases hidratadas existentes en proporción minoritaria. Mediante el análisis de la curva de TGA y el análisis químico realizado al mineral fue posible estimar el porcentaje de caolinita en la arcilla. Así, se observó que la pérdida en masa debido a la deshidroxilación de la arcilla (6\%), es menor que la pérdida en masa de un caolín de composición ideal (14\%). Las pérdidas en masa observadas indican un contenido aproximado del $43 \%$ de caolinita en el mineral, a reserva de la presencia de otros filosilicatos en éste. En el pico observado entre $850-950{ }^{\circ} \mathrm{C}$, dado su anchura, probablemente estén englobados varios eventos, como la descomposición de la fase dolomita y la combustión de materia orgánica. El pico exotérmico observado a $\sim 980^{\circ} \mathrm{C}$, corresponde a la transición de metacaolinita a mullita. Dado el bajo contenido de hierro presente en el mineral, no se observó ningún evento térmico asociado a la descomposición de la limonita.

El tratamiento térmico de la muestra trae consigo una importante variación en las propiedades ópticas del caolín en el intervalo de $800{ }^{\circ} \mathrm{C}$ (CIE Ganz82 = -32,5) a 


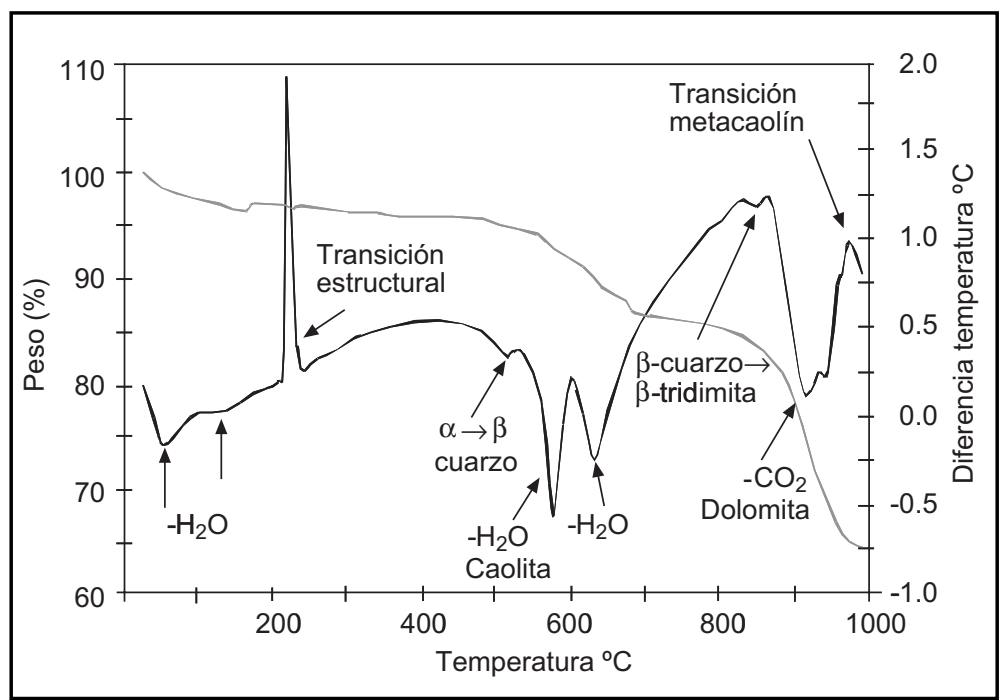

Figura 7. Termograma TGA/DTA del caolín.

Tabla 3

Fases cristalinas detectadas por XRD después del tratamiento térmico del mineral.

\begin{tabular}{|c|c|c|}
\hline Temperatura de calcinación $\left({ }^{\circ} \mathbf{C}\right)$ & Blanqueado CIE Ganz 82 & Fase cristalina detectada por difracción de rayos $\mathbf{X}$ \\
\hline 25 & -23 & caolinita, cuarzo, cristobalita, tridimita \\
\hline 800 & -32.5 & kianita, cuarzo, cristobalita \\
\hline 1.200 & 45.5 & mullita, cuarzo, cristobalita \\
\hline 1.450 & -1.2 & mullita, silimanita, cristobalita \\
\hline
\end{tabular}

$1.450{ }^{\circ} \mathrm{C}$ (CIE Ganz82 = -1,2). Las variaciones de color observado pueden ser asociadas a la formación de diversas fases cristalinas durante el tratamiento térmico. La Tabla 3 muestra las principales fases detectadas por difracción de rayos- $X$ en relación con la temperatura de calcinación. Así, el blanqueo del material observado en el intervalo de $1.200-1.450{ }^{\circ} \mathrm{C}$ es paralelo a la formación de la mullita. De acuerdo a trabajos previos (17), la mullita tiene la capacidad de incorporar cerca del $10-12 \%$ de hierro como $\mathrm{Fe}_{2} \mathrm{O}_{3}$ a través de un mecanismo de formación de solución sólida.

El análisis de dilatometría muestra dos eventos de expansión térmica muy importantes (Figura 8), cuyo cambio energético también se refleja en el análisis por DTA. El primero corresponde a la transición de $\alpha$ a $\beta$, cuarzo $\left(\sim 573^{\circ} \mathrm{C}\right)$, y el segundo ocurre entre 110 y $240^{\circ} \mathrm{C}$, el cual engloba la transición de la forma $\alpha$ a $\beta$ de la tridimita y de la cristobalita (18-20) (Figura 8). El segundo evento, causado principalmente por la cristobalita, conlleva una expansión térmica mayor respecto al primero, aunque ocurre de una manera menos brusca. Mientras que el coeficiente de dilatación lineal en la zona de transición de $\alpha$ a $\beta$ cuarzo es de $92,1 \times 10^{-7}{ }^{\circ} \mathrm{C}^{-1}$, en la zona de transición de $\alpha$ a $\beta$ cristobalita es de $158 \times 10^{-7}{ }^{\circ} \mathrm{C}^{-1}$. Lo anterior implica que si el caolín se destinara a la fabricación de cuerpo cerámico, éste mostraría una gran contracción entre 110 y $240^{\circ} \mathrm{C}$ durante el enfriamiento, por

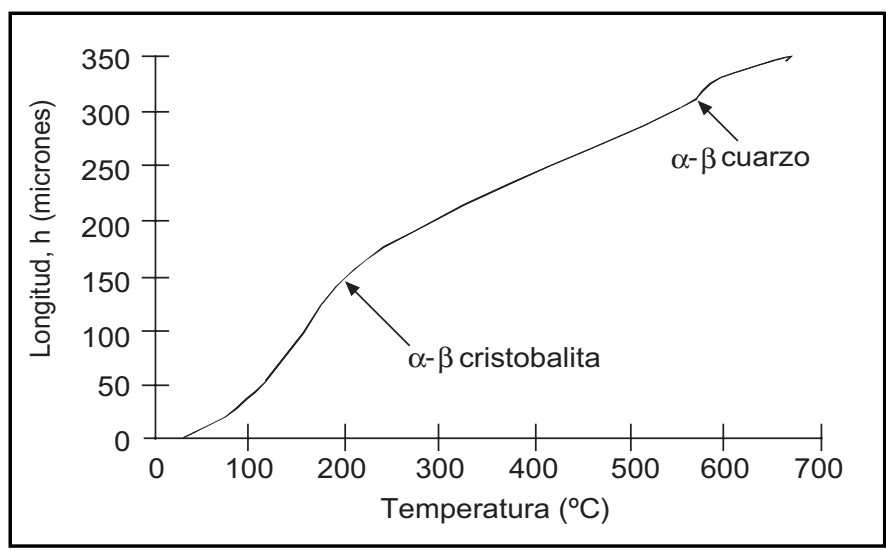

Figura 8. Análisis dilatométrico del caolín. 
lo que deberían de tomarse cuidados especiales durante este proceso, ya que un enfriamiento brusco provocaría la ruptura de las piezas. Por el contrario, la presencia de las fases de alta temperatura del $\mathrm{SiO}_{2}$, unido al vidrio existente en el caolín, favorecería el abrasamiento del clínker, especialmente del clínker blanco. Por lo anterior, el material de Villa de Reyes es más susceptible a ser empleado preferentemente en la industria cementera. Además, el bajo contenido de álcalis hace al material adecuado para su uso en la fabricación de cemento bajo en álcalis. Un tratamiento posterior beneficiamiento del caolín puede que lo hiciese factible para su empleo en la elaboración de cemento blanco, ya que es posible remover una cantidad importante del hierro presente en la muestra. Se recomienda realizar -como trabajo futurouna investigación que involucre los fenómenos de formación de solución sólida, como ocurre durante el proceso de blanqueado del caolín e investigar la forma de emplearlo en la elaboración del cemento blanco sin tener que someterlo a procesos previos de remoción (química) de los elementos cromóforos.

\section{CONCLUSIONES}

En el presente trabajo se ha realizado la caracterización mineralógica del caolín de Villa de Reyes, región cercana a San Luis Potosí (México). La arcilla está constituida principalmente por caolinita, aunque se detectaron otros minerales en pequeñas proporciones (cuarzo, cristobalita, tridimita y dolomita). La presencia de cuarzo en el mineral no es de extrañar dada la geología de la región de donde fue obtenido el material. La presencia de algunos minerales tales como feldespato y hematita, no detectados por difracción de rayos- $\mathrm{X}$, fue observada por microscopía óptica. Mediante la técnica de XANES fue posible determinar que una parte del hierro presente en la muestra se encuentra en forma de limonita $[\mathrm{FeO}(\mathrm{OH})]$. La limonita, que corresponde a la parte mayoritaria del Fe en el caolín (70\%), puede ser removida del mineral mediante un proceso de lixiviación, experimentando éste un proceso de blanqueo. Otro proceso de blanqueo del mineral es posible utilizando un tratamiento térmico conducente a la formación de mullita, lo que conlleva al blanqueo del mineral por incorporación de Fe en ésta mediante un mecanismo de solución sólida en el intervalo de temperaturas de 1.200-1.450 C. Los fenómenos de expansión térmica a baja temperatura experimentados por el caolín de Villa de Reyes, provocados principalmente por la presencia de cristobalita, lo hacen poco recomendable para su uso en cerámica tradicional. Sin embargo, la presencia de las fases de alta temperatura del $\mathrm{SiO}_{2}$ como cristobalita, tridimita y vidrio, además de su bajo contenido en álcalis, lo hacen más susceptible a su empleo como materia prima en la industria cementera.

\section{AGRADECIMIENTOS}

Los autores agradecen a (SEP-2003-C02-42910), SEMARNAT 2004 C01-394 y PAICYT-UANL (CA1248-06, CA1265-06 and CA1270-06) por el apoyo financiero brindado a través de proyectos de investigación. Los autores también desean expresar su agradecimiento al Dr. George Meitzner The University of Texas at El Paso por su ayuda en las mediciones de XANES en el Stanford Synchrotron Radiation Laboratory (SSRL), así como a los ingenieros Javier Vázquez y Homero Ramírez de CEMEX por el soporte económico al proyecto y por haber proporcionado las muestras de caolín para este estudio.

\section{BIBLIOGRAFÍA / BIBLIOGRAPHY}

(1) Murray, H. H.: "Traditional and new applications for kaolin, smectite, and palygorskite: a general overview", Applied Clay Science, vol. 17 (2000), pp. 207-221.

(2) Leite, J. Y. P.; Veras, M. M.; Santos, E. P.; Lima, R. F. S.; Paulo, J. B. A.; Pinheiro, M.: "Technological characterization of kaolin tailing from small-scale mining in RN and PB states-Brazil", Minerals Engineering, vol. 20 (2007), pp. 959-961.

(3) Nguyen, L. D.; Loridant, S.; Launay, H.; Pigamo, A.; Dubois, J. L.; Millet, J. M. M.: "Study of new catalysts based on vanadium oxide supported on mesoporous silica for the partial oxidation of methane to formaldehyde: Catalytic properties and reaction mechanism", Journal of Catalysis, vol. 237 (2006), pp. 38-48.

(4) Siddiquia, M. A.; Ahmeda, Z. A.; Saleemi, A.: "Evaluation of Swat kaolin deposits of Pakistan for industrial uses", Applied Clay Science, vol. 29 (2005), pp. 55-72.

(5) Galindo, R.: "Pastas y Vidriados en la Fabricación de Pavimentos y Revestimientos Cerámicos", Faenza Editrice Ibérica, S.L., España (1994), p. 251.

(6) Toro, L.; Paponetti, A. M.; Passariello, B.: "Proceso Per la Rimozione del Ferro da Concentrati di Caolino, Quarzo ed Altri Materiali di Interese Industriale", Italian Patent, no 217070 A/90 (1990).

(7) Newns, A.; Pascoe, R.D.: "Influence of path length and slurry velocity on the removal of iron from kaolin using a high gradient magnetic separator", Minerals Engineering, vol. 15 (2002), pp. 465-467.

(8) Kuzev, L. V.; Seksenov, S. G.; Kuzeva, R. I.: "Vibration attrition pulverizing. Changing Scopes in Mineral Processing", Proceedings of the $6^{\text {th }}$ International Mineral Processing Symposium, Kusadasi, Turk., sept. 24-26 (1996), pp. 37-39. 
(9) Torres-Martínez, L. M.; Rodríguez-Lugo, Rodríguez Lugo, V.; Rubio, E. L.; Castaño, V. M.: "Synthesis of Silicon Carbide from Rice Husk", Journal of Environment and Pollution, vol. 18 (2002), pp. 377-378.

(10) Behl, S.; Willis, M. J.; Young, R. H.: "Colored titaniferous pigment, obtained by purifying kaolin, for paper coating", Engelhard Corporation, USA, PCT Int. Appl. (1996), p. 27.

(11) Andrews, R. W.; Greenhill, D. A.; Golley, C. R. L.; May, A. A.: "A method of treating an aqueous suspension of kaolin", Imerys Pigments, Inc., USA, PCT Int. Appl. (2000), p. 33.

(12) González, J. A.; Del C. Ruiz, Ruiz, M. del C.: "Bleaching of kaolins and clays by chlorination of iron and titanium", Applied Clay Science, vol. 33 (2006), pp. 219-229.

(13) Veglio, F.; Passariello, B.; Toro, L.; Marabini, A. M.: "Development of a bleaching process for a kaolin of industrial interest by oxalic, ascorbic, and sulfuric acids: preliminary study using statistical methods of experimental design", Industrial \& Engineering Chemistry Research, vol. 35 (1996), pp. 1680-1687.

(14) Cameselle, C.; Núñez, M. J.; Lema, J. M.: "Leaching of kaolin iron-oxides with organic acids", J. Chem Tech. Biotechnol., vol. 70 (1997), pp. 349-354.

(15) Ambikadevi, V. R.; Lalithambika, M.: "Effect of organic acid on ferric iron removal from iron-stained kaolinite", Applied Clay Science, vol. 16 (2000), pp. 133-145.

(16) Norton, F. H.: "Cerámica Fina: Tecnología y Aplicaciones", Ediciones Omega, S.A., Barcelona (1975), p. 44.

(17) Murthy, K.: "X-ray study of the solid solution of $\mathrm{TiO}_{2}, \mathrm{Fe}_{2} \mathrm{O}_{3}$ and $\mathrm{Cr}_{2} \mathrm{O}_{3}$ in mullite $\left(3 \mathrm{Al}_{2} \mathrm{O}_{3} \cdot \mathrm{SiO}_{2}\right)$ ", Journal of the American Ceramic Society, vol. 43 (1960), pp. 267-274.

(18) McNamara, E.: "Introduction to Ceramics", Ceramics, vol II, The Pennsylvania State College, USA (1947), pp. 79-96.

(19) Richardson, D.: "Modern Ceramic Engineering, Properties, Processing and Use in Desing", Marcel Dekker Inc., USA (1982), pp. 1920.

(20) Lozano, D.: "Dilatometría, aplicación en la cerámica tradicional", México, (2000), pp. 33-42. 